\title{
Turbulent jets issuing from the rectangular nozzle with a rectangular notch at the midspan
}

\author{
S. Fujita ${ }^{1}$, T. Harima ${ }^{1} \&$ H. Osaka ${ }^{2}$ \\ ${ }^{1}$ Department of Mechanical and Electrical Engineering, \\ Tokuyama College of Technology, Japan \\ ${ }^{2}$ Emeritus Professor of Yamaguchi University, Japan
}

\begin{abstract}
The mean velocity field of turbulent air jets issuing from a rectangular nozzle $(A R$ : Aspect Ratio=12.5) with a rectangular notch at the midspan have been investigated experimentally. The four notch aspect ratios $(N A R)$ of the rectangular notch perpendicular to the rectangular nozzle axis are $2.5,7.5,12.5$ and 165 . The purpose of this study is to examine the possibility of manipulating the mean velocity field of the rectangular jet using the rectangular notch as a passive disturbance. In this experiment, it is revealed that the inward secondary flow velocities towards the jet centre region on both the rectangular jet axis and the rectangular notch axis in the upstream region are found in all NAR cases, and both the magnitude and the direction of the secondary flow velocity depend on the value of $N A R$. Furthermore, the potential core length of $N A R=12.5$ takes the largest value in all $N A R$ cases. The half velocity width on the rectangular jet axis takes the minimum value for $N A R=165$.
\end{abstract}

Keywords: rectangular jet, rectangular notch, passive control, mean velocity field, secondary flow, streamwise development.

\section{Introduction}

The rectangular jet is very useful in many cases for which the improvement of the entrainment rate or the promotion of mixing is necessary. However, in the case of the employment of the rectangular jet to the engineering field, the elongation of the jet flow is expected in many industrial cases, for example, washing by multiple parallel jets, the uniformity plating technique, the acid washing process of rolling mill and the air curtain (air shield and ventilating). 
To these technical assignments, the extension of the potential core length seems to be one of the effective methods. So far, Trentacoste and Sforza [1], and Sforza and Stasi [2] classified the flowfield of the rectangular jet into three regions (Potential Core region (PC region), Characteristic Decay region (CD region) and Axisymmetric Decay region (AD region)). Furthermore, Marsters and Fotheringham [3] clarified that the streamwise mean velocity profiles showed the saddle-back shape, which was caused by the inward secondary flow, and the reduction rate of the streamwise velocity on the $x$ axis showed the typical feature in the cases of $A R$ (Aspect Ratio) $\geq 6.44$. From the results mentioned above, it seems that the variation of $A R$ changes PC length and eases the velocity reduction of the $\mathrm{CD}$ region for the rectangular jet. Furthermore, Fujita et al. [4] reported that the PC length of both the finite cruciform jet [5] and the twodimensional jet with a rectangular notch [6] showed larger values for $N A R=12.5$ because of the inward secondary flow on the rectangular jet axis. Quinn and Marsters [7] also reported the promotion of PC length for the finite cruciform jet with finite axis length $(A R=11.97)$.

From these results, it is suspected that an addition of the rectangular notch with $A R=10-15$ perpendicular to the rectangular jet promotes the magnitude of the inward secondary flow towards the jet centre region on the rectangular jet axis, and it extends the PC length significantly.

In this report, the mean velocity field of turbulent air jet issuing from a rectangular nozzle $(A R=12.5)$ with a rectangular notch at the midspan has been investigated experimentally. Four notch aspect ratios $(N A R=2.5,7.5,12.5$ and $165)$ of the rectangular notch perpendicular to the rectangular nozzle axis are used. The streamwise variation of the present mean velocity field is examined. From the results, the possibility of manipulating the rectangular jet using the rectangular notch as a passive disturbance will be discussed.

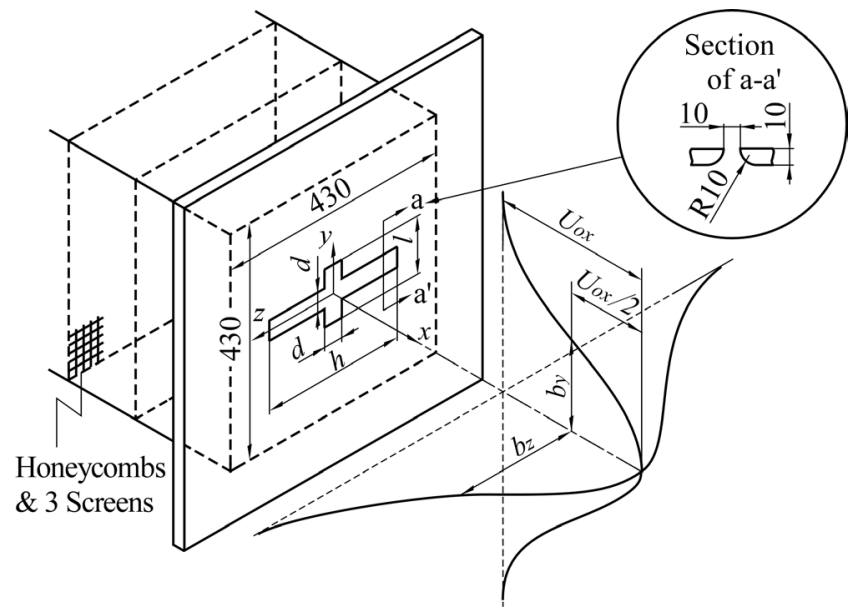

Figure 1: Configuration of the flowfield and the coordinate system. 


\section{Experimental setup and procedure}

The configuration of the flowfield and the coordinate system are presented in fig. 1. The rectangular nozzle with a rectangular notch is installed at the end of the settling chamber. The jet flow facility consists of a turbo fan, a settling chamber fitted with honeycomb and mesh wire screens, and the rectangular nozzle with a rectangular notch. The main axis length $h$ of the rectangular nozzle and the width $d$ are $125 \mathrm{~mm}$ and $10 \mathrm{~mm}(A R=12.5)$, respectively. On the other hand, the main axis length $l$ of the rectangular notch (width $d=10 \mathrm{~mm}$ ) is $25 \mathrm{~mm}$ $(N A R=2.5), 75 \mathrm{~mm}(N A R=7.5)$ and $125 \mathrm{~mm}(N A R=12.5)$, respectively. The rectangular notch length $l$ for $N A R=165$ is $990 \mathrm{~mm}$ and the width $d$ is $6 \mathrm{~mm}$. For the rectangular nozzle, NAR is defined as zero, expediently.

The longitudinal mean velocity was measured using an X-array Hot-Wire Probe $(5 \mu \mathrm{m}$ in diameter, $1 \mathrm{~mm}$ effective length) operated by the linearized constant temperature anemometers (DANTEC), and the spanwise and the lateral mean velocities were measured using a yaw meter. The signals from the anemometers were passed through the low-pass filters and sampled using an A.D. converter at $10 \mathrm{kHz}$. The processing of the signals was made by a personal computer. The acquisition time of the signals was usually 80 seconds.

The exit plane Reynolds number based on the nozzle width $d$ and the exit mean velocity $U_{e}$, was kept constant at $30000(N A R=2.5$ and 7.5), 15000 $(N A R=12.5)$ and $13000 \quad(N A R=165)$ throughout the present experiment, respectively. In this experiment, the uncertainty associated with the longitudinal mean velocity $U$ is estimated at $\pm 3 \%$, which contains the calibration error of the linearized constant temperature anemometers, and the spanwise velocity $V$ and the lateral mean velocity $W$ are estimated at $\pm 6 \%$, which contains the calibration error of the yaw meter.

\section{Experimental results and discussion}

\subsection{Contour plots of longitudinal mean velocity}

The contour plots of $U / U_{e}$ in the upstream region for $N A R=0,2.5,7.5,12.5$ [5] and 165 [6] are shown in figures $2-6$ to indicate the streamwise variation. The dot pattern, which is the same with the outer shape at the exit section of each 3dimensional nozzle, is also shown in each figure.

For $N A R=0$, the contour plot at the section of $x / d=5$ shows almost the rectangular nozzle outer shape, and takes the maximum value at $z / d= \pm 4.5$ on the $z$ axis. At the section of $x / d=10$, the positions of the maximum value of $U / U_{e}$ on the $z$ axis move towards the jet centre region $(z / d= \pm 4.0)$ by the inward secondary flow as will be shown later in fig. 11.

The contour plot at the section of $x / d=2$ for $N A R=2.5$ shows almost the nozzle outer shape, and there is outward prominence of the contour plot parallel to the $y$ axis at $z / d \cong \pm 1.0$. At the section of $x / d=10$, there are five peaks $(z / d \cong 0, \pm 1.5$ and $\pm 3.5)$ having the value of $U / U_{e} \geq 1.0$ on the $z$ axis. There is also outward prominence of the contour plot at $z / d= \pm 1.5$. These prominences at $x / d=2$ and 10 
are produced by the outward secondary flow parallel to the $y$ axis that is caused by collisions between the outward secondary flow and the inward secondary flow at $z / d= \pm 1.0(x / d=2)$ and $z / d= \pm 1.5(x / d=10)$ on the $z$ axis.

Next, the contour plot at the section of $x / d=5$ for $N A R=7.5$ shows the cruciform shape that is almost similar to the nozzle outer shape and takes the maximum values at $z / d= \pm 4$ on the $z$ axis. At the section of $x / d=20$, the width of the contour plot on both the $y$ and $z$ axes become smaller compared to those of $x / d=5$, because of the inward secondary flow on both the $y$ and $z$ axes.

Furthermore, the contour plot at the section of $x / d=5$ for $N A R=12.5$ shows a cruciform outer shape having a good symmetry and takes the maximum values at $y / d=z / d= \pm 4$ on both the $y$ and $z$ axes. At the section of $x / d=30$, the width of

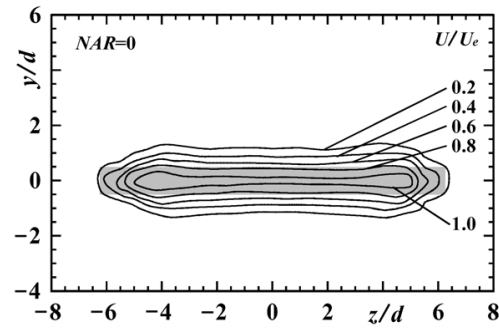

(a) $x / d=5$

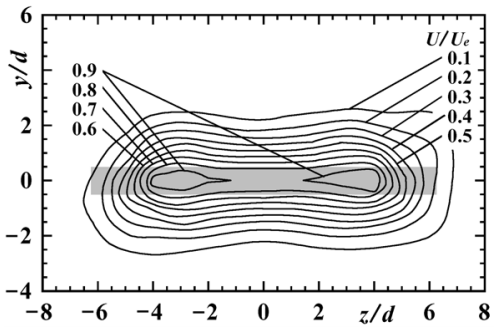

(b) $x / d=10$

Figure 2: Contour plots of longitudinal mean velocity for $N A R=0$.

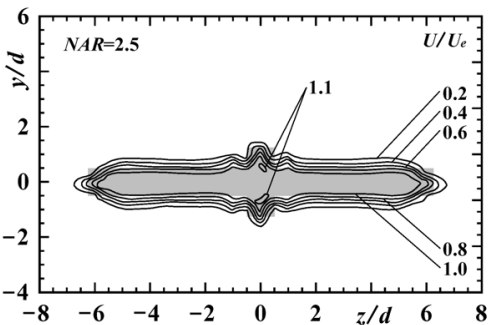

(a) $x / d=2$

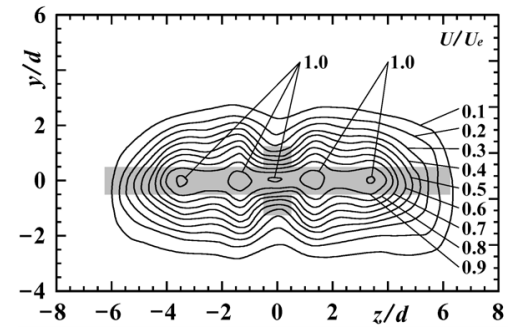

(b) $x / d=10$

Figure 3: Contour plots of longitudinal mean velocity for $N A R=2.5$.

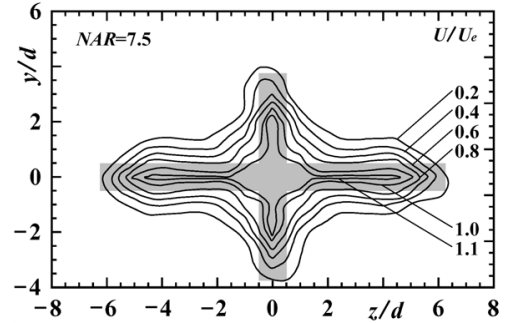

(a) $x / d=5$

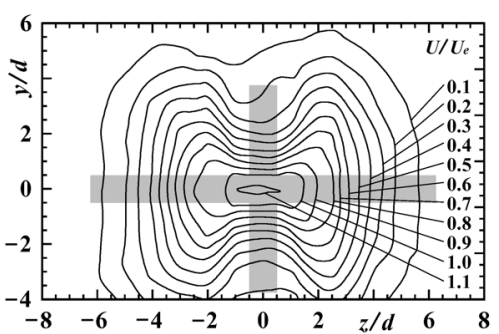

(b) $x / d=20$

Figure 4: Contour plots of longitudinal mean velocity for $N A R=7.5$. 


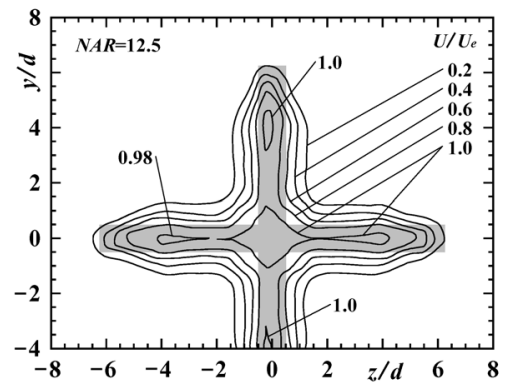

(a) $x / d=5$

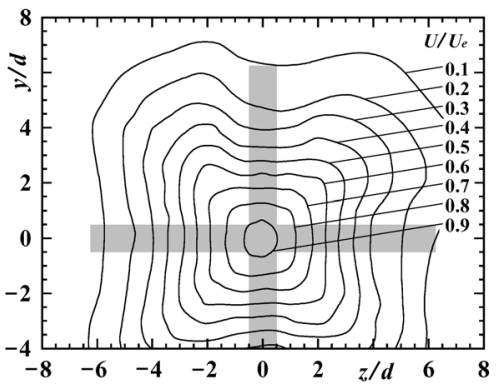

(b) $x / d=30$

Figure 5: $\quad$ Contour plots of longitudinal mean velocity for $N A R=12.5$.

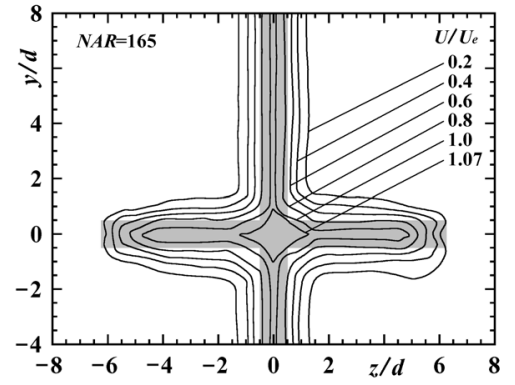

(a) $x / d=5$

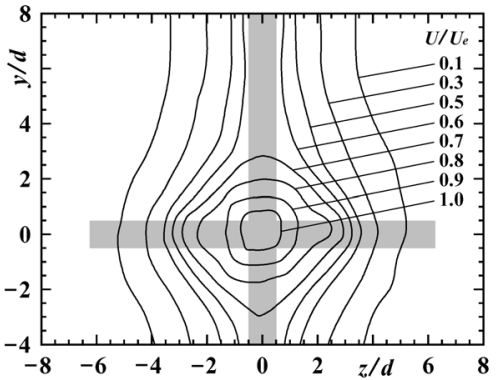

(b) $x / d=15$

Figure 6: Contour plots of longitudinal mean velocity for $N A R=165$.

the contour plot on both the $y$ and $z$ axes become smaller compared to those of $x / d=5$, because of the inward secondary flow on both the $y$ and $z$ axes.

Finally, the contour plot at the section of $x / d=5$ for $N A R=165$ shows a distribution that is close to the nozzle outer shape. At the section of $x / d=15$, the width of the contour plot on the $z$ axis becomes smaller than that of $x / d=5$, because of the inward secondary flow on the $z$ axis.

\subsection{Streamwise variation of the longitudinal mean velocity profiles}

Figures 7-9 show the streamwise variation of the longitudinal mean velocity profiles for $N A R=0,2.5$ and 7.5 on both the $y$ and $z$ axes, respectively. In addition, the profiles on the $z$ axis for $N A R=12.5$ and 165 are also shown in the right hand side and the left hand side in fig. 10, respectively. Here, the symbols used in these figures are the same as $N A R=0$.

At first, the profiles on the $z$ axis (rectangular jet axis) for all $N A R$ will be examined. The profiles at the section of $x / d=5$ for all $N A R$ show a saddle-back shape, which has a peak value near the jet end. These saddle-back shapes are caused by the inward secondary flow on the $z$ axis as will be shown later in fig. 11. At the section of $x / d=10$, the number of peaks $(z / d \geq 0)$ on the $z$ axis is 1 and 3 for $N A R=0$ and 2.5 , respectively. For $N A R \geq 7.5$, there are two peaks. 
66 Fluid Structure Interaction $\mathrm{V}$

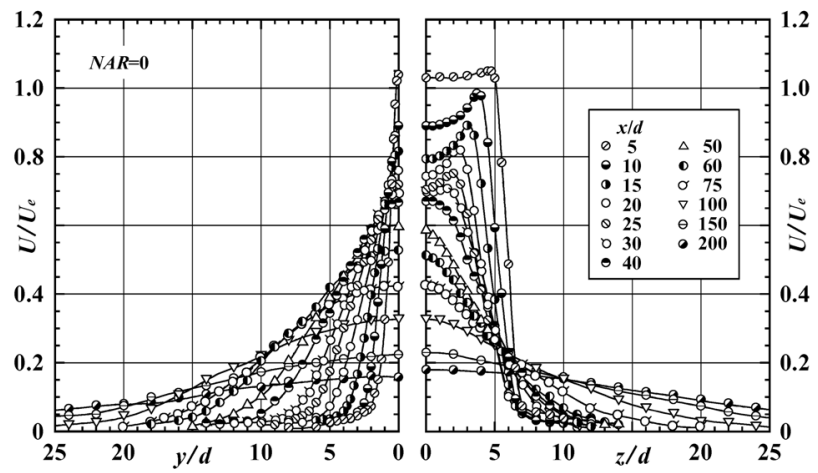

Figure 7: Longitudinal mean velocity profiles for $N A R=0$.

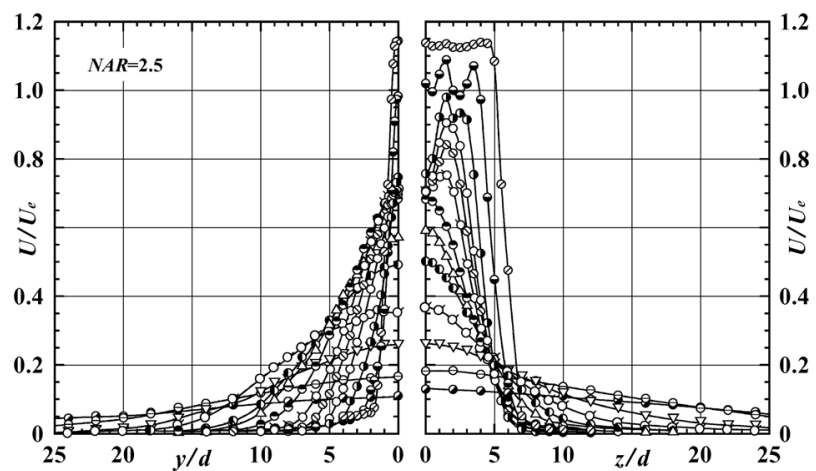

Figure 8: Longitudinal mean velocity profiles for $N A R=2.5$.

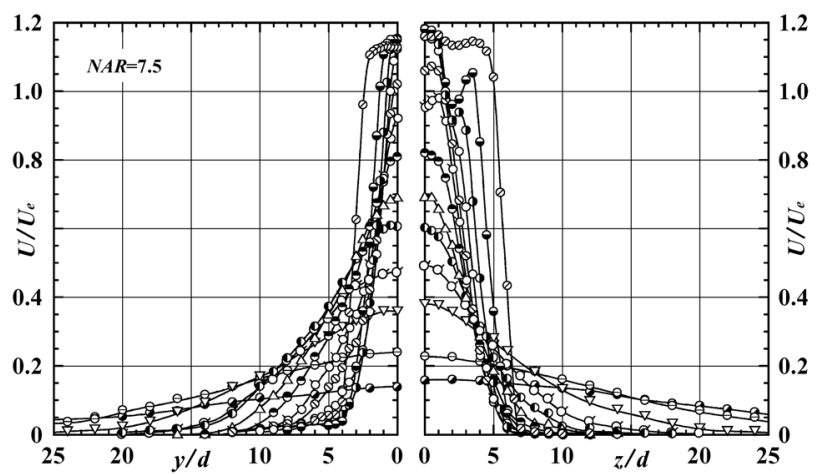

Figure 9: Longitudinal mean velocity profiles for $N A R=7.5$.

On the other hand, the profiles on the $y$ axis for $N A R=0,2.5$ and 7.5 show an ordinary shape which decays monotonously from the maximum value at $y / d=0$. 


\subsection{Streamwise variation of the secondary flow velocity}

Figure 11 presents the profiles of the secondary flow velocity on both the $y$ and $z$ axes at the sections of $x / d=5,10$ and 20 for $N A R=0,2.5,7.5,12.5$ and 165 , respectively.
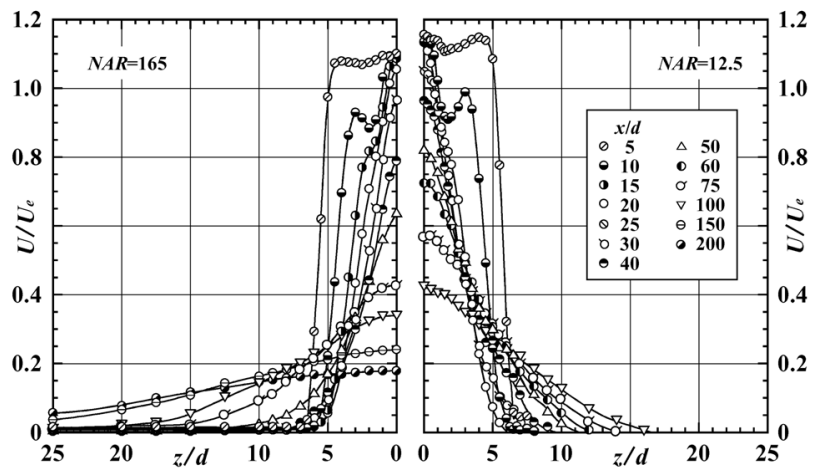

Figure 10: Longitudinal mean velocity profiles for $N A R=12.5$ and 165 .
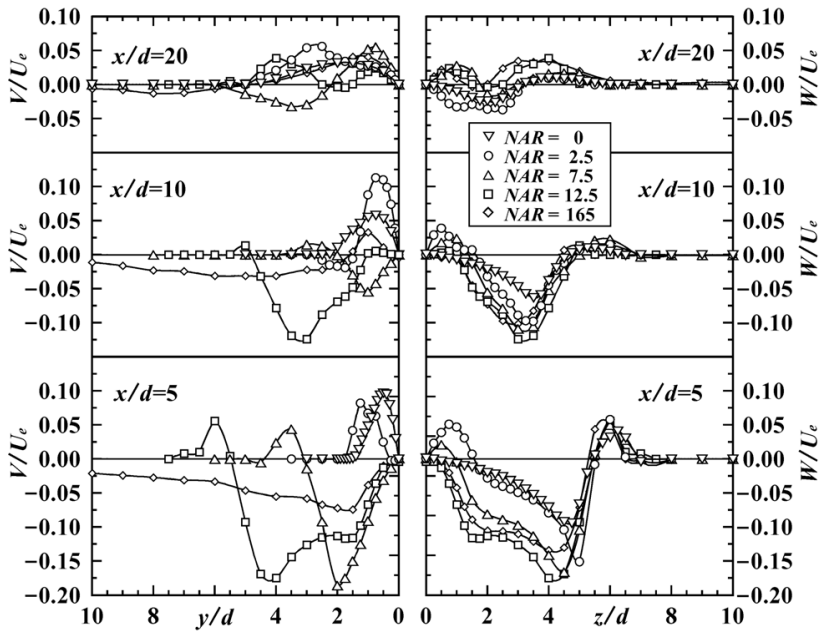

Figure 11: $\quad$ Profiles of the secondary flow velocity on both the $y$ and $z$ axes.

At first, the results on the $z$ axis will be examined. At the section of $x / d=5$, all the profiles show the inward secondary flow velocity toward the jet centre region. And the magnitude of the inward secondary flow for $N A R=12.5$ takes the maximum value. At the section of $x / d=10$, there are also inward secondary flows toward the jet centre for all $N A R$ cases. The magnitude of the inward secondary flow is largest for $N A R=12.5$ [5] and is smallest for $N A R=0$ [5]. This result directly affects the PC length as will be shown in fig. 12. In addition, the 
direction of the secondary flow near the jet centre region of $z / d \leq 1.0$ is outward for $N A R=2.5$ and 7.5. The prominent of the contour plot for $N A R=2.5$ shown in fig. 3-(b), is caused by this outward secondary flow velocity near the jet centre region. Finally, at the section of $x / d=20$, there are still inward secondary flows for $N A R=0,2.5,7.5$ and 12.5 .

Next, the profiles on the $y$ axis will be examined. At the section of $x / d=5$, the inward secondary flows exist for $N A R \geq 2.5$. Here, the inward secondary flows for $N A R=7.5$ and 12.5 show almost the same large values. At the section of $x / d=10$, the inward secondary flow for $N A R=12.5$ shows the maximum value. Finally, at the section of $x / d=20$, there are still the inward secondary flow for $N A R=7.5$ and 12.5 .

From the results mentioned above, the magnitude and the direction of the inward secondary flow vary with the value of $N A R$. Using these features, it seems to be possible to manipulate the PC length of the rectangular jet.

\subsection{Streamwise variation of velocity and length scales}

Figure 12 shows the streamwise variations of the longitudinal mean velocity $U_{o x} / U_{e}$ on the $x$ axis for all $N A R$. Here, the result of the rectangular jet $(A R=10)$ by Sfeir [8] is plotted in the same figure for comparison. All the PC lengths of the rectangular jet with rectangular notch for $N A R \geq 2.5$ take larger values compared with that of the rectangular jet $(N A R=0)$. This reason is explained as that there are the advection transports $\left(W \partial\left(U^{2} / 2\right) / \partial z\right.$ and $\left.V \partial\left(U^{2} / 2\right) / \partial y\right)$ toward the jet centre region of the mean kinetic energy by the inward secondary flow velocity on both the $z$ (the rectangular jet) and $y$ (the rectangular notch) axes. Furthermore, the length of the PC region varies with $N A R$ and takes the largest value for $N A R=12.5$. This reason is explained as that the inward secondary flow for $N A R=12.5$ shows the maximum value on both the $z$ and the $y$ axes as shown in fig. 11. From these results, it seems that the PC length of the rectangular jet can be manipulated by the variation of $N A R$. On the other hand, $U_{o x} / U_{e}$ for $N A R=2.5$ shows the local maximum value in the vicinity of $x / d=34$. This reason is considered as that the mean kinetic energy on the $z$ axis is transported to the jet centre region by the inward secondary flow existing on the $z$ axis at $x / d=20$.

The half velocity widths on both the $y$ and $z$ axes are shown in fig. 13 in order to examine an effect of the value of $N A R$ on the length scales. At first, the values of all half velocity widths $b_{z} / d$ on the $z$ axis decrease from almost the same value of $x / d=2$ and they all take the minimum value at each streamwise section. These decreasing features are caused by the inward secondary flow [5] on the $z$ axis mentioned above. These minimum values of $b_{z} / d$ become smaller with increasing $N A R$, and the $b_{z} / d$ for $N A R=165$ takes the smallest value. From these results, the development of the half velocity width $b_{z} / d$ on the $z$ axis for the rectangular jet seems to be manipulated by the variation of $N A R$. On the other hand, the values of $b_{y} / d$ for $N A R=2.5,7.5$ and 12.5 decrease from each value at the section of $x / d=2$ and take each minimum value at the sections of $x / d=8,20$ and 30 , respectively. 


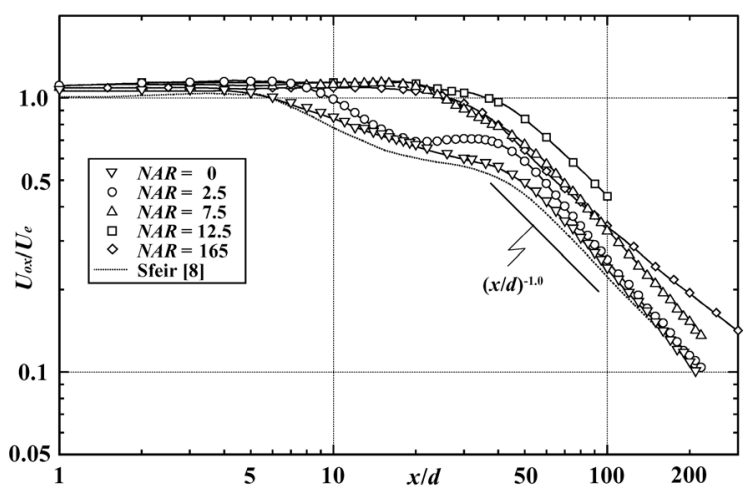

Figure 12: Streamwise variation of velocity scales.

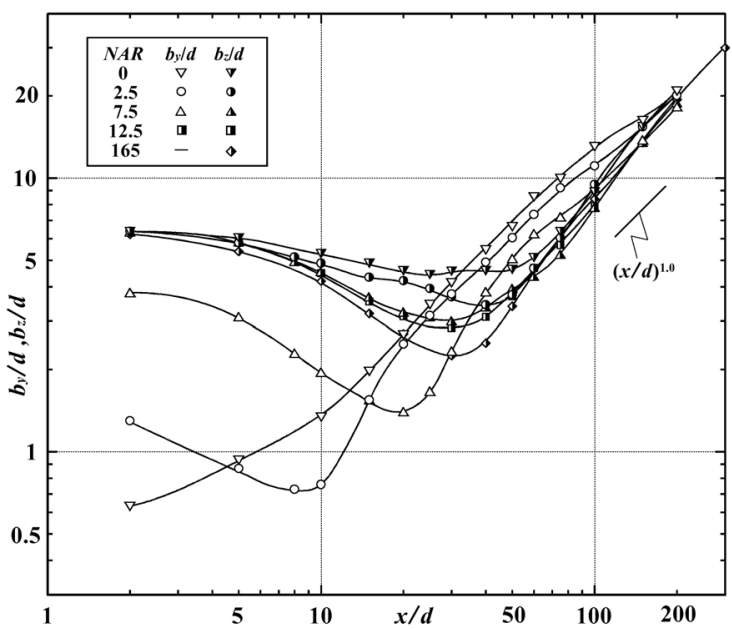

Figure 13: $\quad$ Streamwise variation of length scales.

\section{Conclusions}

The effect of the variation of $N A R$ on the mean velocity fields has been investigated in the turbulent jet issuing from a rectangular nozzle $(A R=12.5)$ with a rectangular notch $(N A R=2.5,7.5,12.5$ and 165$)$ at the midspan, and the results are as follows:

(1) In the upstream region, the mean velocity profiles on the $z$ axis show the saddle-back shape for all $N A R$ cases because of the inward secondary flow velocity toward the jet centre region.

(2) All the PC lengths of the rectangular jet with rectangular notch for $N A R \geq 2.5$ take larger values compared with that of the ordinary rectangular jet. This reason 
is explained as that there are the advection transports toward the jet centre region of the mean kinetic energy by the inward secondary flow velocity on both the $z$ (the rectangular jet) and $y$ (the rectangular notch) axes in the upstream region. And the length of the $\mathrm{PC}$ region varies with $N A R$ and takes the largest value for $N A R=12.5$. Therefore, the PC length of the rectangular jet can be manipulated by the variation of $N A R$.

(3) The half velocity width $b_{z} / d$ on the $z$ axis for all $N A R$ take each minimum value in the upstream region because of the inward secondary flow velocity toward the jet centre region, and the $b_{z} / d$ takes the minimum value for $N A R=165$. Therefore, the development of the half velocity width $b_{z} / d$ on the $z$ axis for the rectangular jet seems to be manipulated by the variation of $N A R$.

\section{References}

[1] Trentacoste, N. and Sforza, P.M., Further experimental results for threedimensional free jets. AIAA Journal, 5(5), pp. 885-891, 1967.

[2] Sforza, P.M. and Stasi, W., Heated three-dimensional turbulent jets. Transaction of the ASME, Journal of Heat Transfer, 101(2), pp. 353-358, 1979.

[3] Marsters, G.F. and Fotheringham, J., The influence of aspect ratio on incompressible, turbulent flows from rectangular slots. Aeronautical Quarterly, 31(4), pp. 285-305, 1980.

[4] Fujita, S. et al., Turbulent jets issuing from rectangular nozzle with a rectangular notch at the midspan (mean velocity field). Transactions of the Japan Society of Mechanical Engineers, Series B, 72(717), pp. 1159-1165, 2006.

[5] Fujita, S. and Osaka, H., Three-dimensional jets issuing from cruciform nozzles having four aspect ratios (1st Report. Mean Flow Properties). Transactions of the Japan Society of Mechanical Engineers, Series B, 53(488), pp. 1142-1149, 1987.

[6] Fujita, S. et al., Turbulent jet issuing from a quasi two-dimensional nozzle with a rectangular notch at the midspan (streamwise variation of mean velocity field). Transactions of the Japan Society of Mechanical Engineers, Series B, 65(631), pp. 905-911, 1999.

[7] Quinn, W.R. and Marsters, G.F., Upstream influence on turbulent jet flows from cruciform nozzles. Aeronautical Journal, 89(882), pp. 55-58, 1985.

[8] Sfeir, A.A., Investigation of three-dimensional turbulent rectangular jets. AIAA Journal, 17(10), pp. 1055-1060, 1979. 